cefmetazole, fluoroquinolones, gentamicin, and trimethoprimsulfamethoxazole after $\mathrm{T} 14$ and $\mathrm{C} 10$ at week 2 returned to the basal state at week 8 and 1 year. Although body weight and BMI slightly increased, there were significant improvements in metabolic parameters with a decrease in insulin resistance, triglycerides and low-density lipoprotein, and an increase in high-density lipoprotein. Overall, there was no significant change in the prevalence of metabolic syndrome at week 8 and 1 year, after T14, C10, and BQ10. (figure 1)

Conclusions Eradication of $H$. pylori infection has minimal disruption of the microbiota, no effect on antibiotic resistance of E. coli and some positive effects on metabolic parameters. These collectively lend support to the long-term safety of $H$. pylori eradication therapy

\section{IDDF2019-ABS-0219 EFFICACY AND SAFETY OF ADDITIONAL AUTOLOGOUS PLATELET RICH STROMA IN TRANSANAL MUCOSAL ADVANCEMENT FLAP REPAIR OF TRANSSPHINCTERIC CRYPTOGLANDULAR PERIANAL FISTULAS}

\footnotetext{
${ }^{1}$ Jeanine Arkenbosch*, ${ }^{2}$ Oddeke van Ruler, ${ }^{3}$ Jeroen Stevens, ${ }^{2}$ Eelco de Graaf, ${ }^{2}$ Ruud Schouten, ${ }^{1}$ Annemarie de Vries, 'Janneke van der Woude. ${ }^{1}$ Department of Gastroenterology and Hepatology, Erasmus Medical Center, Rotterdam, Netherlands; ${ }^{2}$ Department of Surgery, IJsselland Hospital, Capelle a/d IJssel, Netherlands; ${ }^{3}$ Department of Plastic and Reconstructive Surgery, Bergman Clinic, The Hague, Netherlands

\subsection{6/gutjnl-2019-IDDFabstracts. 10}

Background Treatment of transsphincteric cryptoglandular perianal fistulas is challenging and associated with high recurrence rates. Transanal Advancement Flap Repair (TAFR) fails in almost one of every three patients, probably due to persistent chronic inflammation. Autologous Platelet-rich Stroma (PRS), platelet-rich plasma (PRP) combined with progenitor cells from autologous stromal vascular fraction (SVF), obtained from liposuction, could suppress chronic inflammation and improve success rates in TAFR. This study aimed to assess the feasibility, safety and efficacy of additional injection of autologous during TAFR of transsphincteric cryptoglandular fistulas.

Methods 22 patients with transsphincteric cryptoglandular fistulas who underwent TAFR between December 2017 and October 2018 were prospectively included after informed consent. All patients underwent standardized TAFR and standardized preparation of autologous PRS. Inclusion criteria were transsphincteric fistulas with only one internal opening (or a second one very close by) and absence of pelvic abscess. Clinical healing was defined as the absence of symptoms and closure of the external opening at physical examination.
Radiographic closure was defined as complete closure on postoperative MRI.

Results This study included 22 consecutive patients (10:12 male: female; median age 44 years (IQR 33-55). Follow-up data of at least 6 months was available for 18 of patients to date. All patients had one or more previous operations ranging from seton drainage to previous TAFR (3/18) or ligation of the intersphincteric fistula tract (LIFT; 2/18). Clinical healing was reached in 16 out of 18 (88.9\%) patients after a median postoperative follow-up of 3 months (IQR 2.3-5.0). Two of the 18 patients did not show clinical healing at their last consultation at 4 months follow-up. Of the available 15 MRIs to date (1 is pending), 14 showed radiographic closure (93.3\%). Postoperative complications encompassed thrombosis $(n=1)$ and reoperation due to rebleeding $(n=1)$.

Conclusions In 18 patients with transsphincteric cryptoglandular fistula treated with the addition of autologous PRS during TAFR, 93\% (14/15) showed complete radiographic closure. The addition of autologous PRS appears to be feasible, safe and highly promising due to high success rates. More studies are needed to determine the exact impact.

\section{Clinical Hepatology}

\section{IDDF2019-ABS-0073 A SIMPLIFIED PROGNOSTIC MODEL TO PREDICT MORTALITY IN PATIENTS WITH ACUTE VARICEAL BLEEDING: MULTICENTER STUDY RESULTS}

Sakkarin Chirapongsathorn*, Kuntapon Akkarachinores, Amnart Chaipresert, Pawinee Saybungkla. Division of Gastroenterology and Hepatology, Phramongkutklao Hospital, College of Medicine, Thailand

\subsection{6/gutjnl-2019-IDDFabstracts.11}

Background Acute variceal bleeding (AVB) is a serious complication associated with high mortality. The aim of our study was to investigate mortality predictors and develop a new simple prognostic model using easily verified factors at admission in AVB patient.

Methods Between October 2012 and September 2018, 1,144 consecutive patients with AVB from Phramongkutklao hospital in Bangkok and Maharat Nakhon Sri Thummarat hospital in Nakhon Sri Thummarat were included. A simplified prognostic model was developed using multiple logistic regression after identifying significant predictors of 6-week mortality. Mortality prediction accuracy was assessed with area under the receiver operating characteristic (AUROC) curve. We compared the

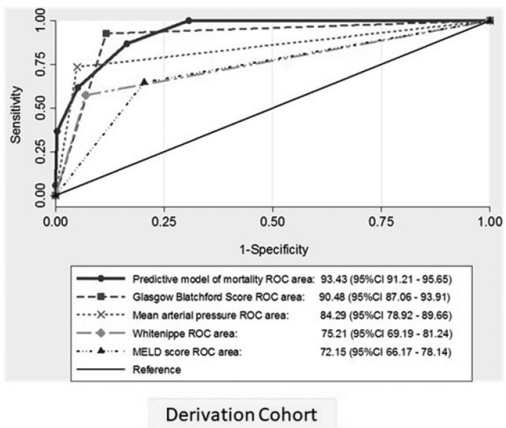

Model to predict mortality in 6 weeks

Area under ROC curve (AUROC) $\mathbf{0 . 9 3 4 3}$

- Sensitivity $89.04 \%(95 \% \mathrm{Cl}: 86.48-91.60)$

- Specificity $85.34 \%(95 \% \mathrm{Cl}: 82.44-84.44)$

Abstract IDDF2019-ABS-0073 Figure 1 Compare model to others predictors for mortality in 6 weeks 